\title{
Teeth are for chewing: a critical review of the conceptualisation and ethics of a controversial intraoral weight-loss device
}

\author{
Cat Pausé, ${ }^{\star 1}$ Tara G. McAllister, ${ }^{2}$ Aimee B. Simpson, ${ }^{2}$ Rebekah Graham, ${ }^{3}$ Laura Calloway, ${ }_{1}^{4}$ Ashlea Gillon, ${ }_{1}^{2}$ Sian Halcrow, ${ }^{5}$ \\ Rhys Jones, ${ }^{2}$ Samantha Keene, ${ }_{1}^{6}$ Andrea LaMarre, ${ }_{1}^{7}$ George Parker, ${ }_{1}^{6}$ Darren Powell, ${ }^{2}$ Toby Santa Maria, ${ }_{1}^{8}$ Brooke Tohiariki, ${ }_{1}^{5}$ \\ Emma Tumilty, ${ }^{9}$ Callie Vandewiele, ${ }^{2}$ Alison Watkins ${ }^{10,11}$ and Cassie Withey-Rila ${ }^{5}$
}

\section{Key points}

Provides a critique of a recent publication in the British Dental Journal.

Highlights ethical concerns and issues of bias present within research of the DentalSlim Diet Control.
Emphasises the role that white supremacy, patriarchy, and anti-fat attitudes, play in the development and evaluation of the DentalSlim Diet Control.

\begin{abstract}
We are a diverse collective of researchers who are committed to improving the health and wellbeing of marginalised individuals. This article is a response to, and critique of, the DentalSlim Diet Control research. This device revises a controversial 1970s weight-loss technology connected to poor health outcomes, which is indicative of a culture that consistently promotes harm to fat and other marginalised communities.

We address the historical context in which unruly bodies, particularly fat, and Indigenous bodies have been the site of unethical investigation conducted under the auspices of medical research. Existence outside the normative white, male, cis physical ideal demands regulation, and disciplinary measures. We demonstrate how Brunton et al.'s research is underpinned by anti-fat attitudes and assumptions which impose this punitive physical intervention onto healthy people in a way that should not be acceptable in medical research.

Further, we address a range of harms, giving attention to Māori and to individuals with eating disorders, along with issues of research integrity. We argue that no ethics committee should have approved this research, no academic journal should have published it, and no member of the dental and medical community should promote or prescribe this device.
\end{abstract}

On 28 June 2021, the University of Otago published a press release regarding the development of an intraoral weight-loss device, the DentalSlim Diet Control. Results from a study of the device are published in the British Dental Journal. ${ }^{1}$ The study was led by Professor Paul Brunton, Pro-Vice Chancellor of Health Sciences at the University of Otago.

'Massey University, Palmerston North, New Zealand; 'University of Auckland, Auckland, New Zealand; 'PParents of Vision Impaired NZ, Hamilton, New Zealand; ${ }^{4}$ ndiana University Bloomington, Bloomington, USA; ${ }^{5}$ University of Otago, Dunedin, New Zealand; ' 6 Victoria University of Wellington, Wellington, New Zealand; ${ }^{7}$ Massey University, Auckland, New Zealand; ${ }^{8}$ Michigan State University, East Lansing, USA; ' Deakin University, Geelong, Australia; ${ }^{10}$ University of Canterbury, Christchurch, New Zealand; "Auckland University of Technology, Auckland, New Zealand. *Correspondence to: Cat Pausé

Email address: c.pause@massey.ac.nz

Accepted 30 September 2021

https://doi.org/10.1038/s41415-021-3680-x
The press release and associated study attracted substantive online scrutiny and global criticism.

This paper is a response to, and critique of, the research conducted by Brunton et al. ${ }^{1}$ We address the underlying assumptions and biases of their intervention, and point to how they are harmful and dangerous, paying particular attention to their impact on fat people, Māori and people with eating disorders (EDs). We are a diverse multidisciplinary group of scholars who came together through Twitter in our shared interest and outrage. We purposely do not use 'overweight' and 'obesity' and instead use fat, the fat body and fat people, in order to avoid pathologising and further stigmatising fat people. ${ }^{2}$ We outline failures in the ethical review process of this research, including issues of research integrity in the reporting of findings.

Medical researchers and practitioners often conflate appearance with health, especially in relation to body size and fatness. Fat patients are frequently perceived by physicians as less compliant and ultimately unruly. ${ }^{3}$ Fat stigma is heavily intertwined with racism. ${ }^{4}$ Healthism, policing and pathologising of Māori bodies has a long history in Aotearoa New Zealand (Aotearoa) and is grounded in white supremacy and colonialism. ${ }^{5,6}$ Fat Māori experience increased barriers to accessing healthcare, receive poorer-quality care, are less likely to be referred to specialist services and are less likely to receive timely diagnosis of life-threatening or life-altering conditions. ${ }^{7}$

For decades, doctors have been (re) developing technologies that eliminate fatness, including techniques such as jaw-wiring - an intervention initially devised to stabilise and immobilise the jaw following trauma and/ or surgery. Jaw-wiring rose to popularity in the 1970 s as a weight-loss technique, and it was alluring as it provided a non-surgical 
solution to fatness that could be easily fitted in a dental office which, while in place, induced comparable rates of weight loss to bariatric surgery. ${ }^{8}$ As a standalone intervention, jawwiring eventually fell out of favour due to universal weight regain after the device was removed. However, it continued to be recommended for integration into wider weight-loss programmes, such as preparation for patients undergoing bariatric surgery. ${ }^{9}$

Through jaw immobilisation, physicians ensure patient compliance with common pre-surgery stipulations, such as a strict, lowcalorie liquid diet to induce mandatory weight loss. ${ }^{10}$ In this way, jaw immobilisation dually functions as a form of bodily control that prevents the intake of substantive nutrition and a tool to enhance the efficacy of other weightloss methods. This highlights the way that such devices enforce control and punishment - staples of all weight-loss initiatives - over fatness and fat bodies. ${ }^{11}$ Indeed, the continued resurfacing of these weight-loss methods is indicative of a broader cultural landscape which actively encourages the elimination of fatness and fat people through restrictive dietary practices.

Across the broader cultural landscape, it is believed that fat people are responsible for their fatness, and that they knowingly and willingly engage in behaviours that make them fat. ${ }^{11}$ As fat people are deemed morally culpable for their fatness, shame and stigma act as a powerful form of discipline. ${ }^{11}$ Shame occurs as a result of social stigma and marginalisation, and can drastically impact health, illness and health-related behaviours. ${ }^{12}$ It is well known that fat people often experience stigma and marginalisation as a result of their body size. ${ }^{13,14}$ Stigma experienced by fat people often results in negative health, emotional and relational consequences as it can prevent individuals from fully engaging in their lives. ${ }^{11,13}$ Shame associated with fatness is also disproportionately experienced by people faced with multiple axes of oppression. As shame and stigma are both powerful social determinants of health, it is striking that the analysis by Brunton et al. ${ }^{1}$ fails to adequately consider the ways that fat stigma and shame contribute to, and exacerbate, poor health outcomes for fat people.

The physically restrictive nature of DentalSlim reflects the dominant narrative that fatness is attributable to a lack of selfrestraint, and therefore fat bodies require external control. The inventors of DentalSlim suggest that the device is appropriate for those for whom 'dietary adherence is very shortterm, ${ }^{1}$ emphasising fat people's perceived lack of willpower. Another example of this is the description of a participant who disclosed they had 'cheated' by consuming melted chocolate and fizzy drinks. The authors conclude that this participant's behaviour was 'not surprising' as 'obese patients usually have an addictive personality and an impulsivity for sugary food and suffer from binge-eating disorders.' ${ }^{1}$ Words such as 'cheated', 'addictive' and 'impulsivity' are illustrative of the underlying assumption that fatness is a behavioural problem relating to poor self-control and regulation. This type of broad-brush, reductionist depiction of fat people as inherently 'out of control' provides moral justification for restricting their eating. ${ }^{11}$ The language utilised by Brunton et al. ${ }^{1}$ towards their fat participants reinforces a deficit view of fat bodies, perpetuates harmful stereotypes and frames fatness as a shameful state to be avoided at all costs.

Brunton et al. ${ }^{1}$ acknowledge that 'weight was the main reason for experiencing public distress, such as being discriminated against by others' and that participants' concerns relating to public distress remained 'unchanged' following the wearing of the device. ${ }^{1}$ Additionally, after 24 hours, participants described feeling embarrassed, self-conscious and that, in general, their life was less satisfying. Such findings necessitate attending to the pervasive negative effects of fat stigma on the lives of their participants. Yet, the researchers go on to say that participants became accustomed to wearing the device. Indeed, becoming accustomed to a device, despite experiencing physical and psychological discomfort, is indicative of the ways in which fat people feel, and are made to feel, that they must atone for their fat bodies and behaviour. ${ }^{11}$ However, the authors neglect to discuss fat stigma, atonement, or shame, concluding instead that the device is a 'tolerable' solution and one which 'motivated the subjects to continue on their weight-loss journey. ${ }^{1}$ These conclusions do not accurately reflect the evidence in participant experiences with the device. Instead, the conclusions are centred on the authors' own ideas and biases regarding the regulation of fat bodies and their assumptions regarding the importance of weight loss for improving health outcomes.

The DentalSlim trial recorded minor psychological distress after just one day of restriction. In earlier jaw-wiring studies where participants were immobilised for months, researchers documented 'bouts of severe depression' and attempted suicide. ${ }^{15}$ Yet, in both studies, researchers concluded that the device had improved the quality of life of their participants. Quality of life is deeply connected to food; the myriad ways in which people prepare, eat and share food with others reinforces their shared sense of identity and connection with wider groups. ${ }^{16}$ For Māori, sharing and consuming kai is ritual for spiritual safety and manaakitanga, and is central to building and maintaining relationships with each other and morethan-human relatives. Stripping food back to liquid nutrients undermines these processes' connection. We see examples of this erasure with participants commenting on the isolation they felt at not being able to participate in family dinners and feeling awkward and embarrassed with regards to social functions. ${ }^{1}$ The dismissal of the role of food in relationships, identity and community wellbeing is yet another example of how this research dismisses Indigenous ways of knowing in favour of white biomedical beliefs about the role of food.

As previously discussed, DentalSlim participants reported that the dental device was uncomfortable and reported that life was 'less satisfying' while wearing the device. ${ }^{1}$ These physical and social harms were frequently minimised as participants did not require pain medication, were able to 'tolerate' the device and qualitatively reported they were 'happy with outcome. ${ }^{1}$ Further, the authors describe the device as 'helpful for short-term weight loss,' despite participants regaining weight when the device was removed, thereby indicating its ineffectiveness for long-term health outcomes. The fact that six fat women were willing to tolerate such physical discomfort and social isolation to lose an average of six kilograms over two weeks, and reported happiness with the outcome despite subsequent weight gain, speaks volumes about the negative effects of fat oppression, internalised attitudes to fat bodies and the harm fat people are expected to tolerate in order to lose weight.

Brunton et al. ${ }^{1}$ mention EDs superficially, but do not address the potential risks this device holds in regard to EDs. This omission presents a serious risk to the safety of those who may use this device and reflects incorrect assumptions about who gets EDs, such as the idea that fat people do not develop restrictive EDs. Fatness is not a protective factor against 
disordered and restrictive eating; instead, it often takes longer for fat people to receive accurate diagnosis and treatment. ${ }^{17}$

Additionally, Brunton et al. ${ }^{1}$ conflate fatness and binge eating, and imply that the device could assist people with binge-eating disorder (BED) through weight loss. This framing reflects anti-fat bias in medical settings and fails to address BED as a psychological and behavioural disorder. ${ }^{18}$ Weight-loss oriented approaches for treating BED carry a high likelihood of weight regain and appear less effective at reducing BED symptomatology than approaches that address cognitions and behaviours. ${ }^{19}$ In a world where dieting is normative and weight loss is encouraged at any cost, disordered eating may not be visible. ${ }^{17}$ This leaves people with serious EDs, such as atypical anorexia (often anorexia nervosa without a low BMI), overlooked and undertreated. ${ }^{17}$ People who do not fit the stereotypical (thin, white, ablebodied, heterosexual, cisgender woman) picture of who gets an ED face additional barriers; for instance, there is a lack of awareness throughout the healthcare system about Māori with EDs. ${ }^{20}$

While the device would require a prescription from a medical professional, physicians receive minimal training on $\mathrm{ED}$ recognition, referral and treatment. ${ }^{21}$ They are likely to be unaware of the severity of malnutrition and other health impacts (for example, heart, stomach, bone, blood sugar and other issues) of restriction among people who are not emaciated. Proposing forced dietary restriction as potentially helpful for BED, without 1) clarifying how EDs would be screened for; 2) ensuring pathways to treatment; or 3) at the very least specifying the potential risk for developing or worsening disordered eating, is irresponsible and unethical.

The involvement of the Pro-Vice Chancellor of Health Sciences in this research brings his leadership into question. Universities in Aotearoa, and by extension the researchers they employ, have an obligation to Māori under Te Tiriti o Waitangi. We would question how this research aligns with the University of Otago's espousals of valuing Te Tiriti o Waitangi, also written into the University of Otago's Māori Strategic Framework. This research reinforces fatphobia and racism among medical practitioners and researchers. It re-traumatises people who are fat and who hold identities subject to discrimination and intersecting oppressions. ${ }^{5}$

After enumerating the multiple harms associated with this device, it is now critical to discuss the ethical practices behind the device's study itself. Ethical review should reassure and protect potential participants by vetting research proposals to ensure participant safety, wellbeing and dignity. ${ }^{22,23}$ Ethics committees need to have a clear understanding of the potential benefits and harms of a proposal's methodology in order to determine their ethical acceptability. ${ }^{24}$ Ensuring ethical commitments are met requires an understanding of the researcher's position to, and relationship with, the population they purport to 'help'.

Brunton et al. ${ }^{1}$ claim that their research 'was conducted in full accordance with the World Medical Declaration of Helsinki', yet the general principles of the Declaration of Helsinki are to ensure that the health of participants is always their first consideration; and it is their duty to protect the life, health, dignity and integrity of participants involved in medical research. ${ }^{23}$ However, researchers were allowed to recruit 'healthy' participants into a research study which presented a significant physical intervention onto their bodies with potentially severe psychological risks, including suicide, as highlighted in previous studies of similar devices (jaw-wiring) in the 1970s which they referenced in their work.

In this instance, the review of this research by the Northern B Health and Disability Ethics Committee (NB-HDEC) failed to ensure the safety, wellbeing and dignity of the participants it is tasked with protecting, generating the loss of participant, community and societal trust. We discuss each aspect in turn, beginning with safety and concluding with comment on the integrity of the research.

The minutes of NB-HDEC's review of this project show that harms were barely considered. ${ }^{25}$ Questions focused on the wording of information sheets, questionnaires and a superficial discussion of Māori participation. Harms discussed were related to oral hygiene and the ability to unlock the device in an emergency. Omitted was consideration of EDs, placing any participant with an (unrecognised) $\mathrm{ED}$ at serious risk. Jaw-wiring terminology is used in the ethics application. Elective jaw immobilisation reduces a person's bodily autonomy by restricting their ability to use their body and determine for themselves what goes in it, contributing towards psychological distress, ${ }^{15}$ yet there was no consideration of the significant harms of this technique (discussed earlier in this paper). There was also a lack of consideration given to intersectional oppressions, with the committee only asking for representative numbers of Māori and Pasifika. The approval of this project brings into question the ability of NB-HDEC to keep people, particularly fat people and fat Māori, safe from psychological and physical harm.

The DentalSlim research is based on the flawed assumption that weight loss, through whatever method and no matter the side effects, is a positive and desired outcome for individuals who fall outside socially accepted 'body size' measures. The benefits of pre-surgical weight loss in preparation for bariatric surgery are unknown, ${ }^{26}$ in part due to surgical complications for fat people being measured inconsistently or not at all, ${ }^{27}$ and a lack of methodological rigour in assessments. ${ }^{28}$ As discussed previously, the common practice of equating weight with health, self-control and dietary habits creates a social structure that encourages physicians to police fat patients. The policing of nonnormative bodies is synchronous with the experiences of female, Indigenous, disabled, LGBTQI+ and other marginalised groups within the medical system. The absence of engagement in the ethics of this research is particularly problematic in the Settler-State of Aotearoa where Māori and Pasifika bodies are already devalued by state institutions such as universities and healthcare settings. ${ }^{6}$

Researchers have a duty of care to participants during the research period and into the publication phase. ${ }^{23}$ Throughout their publication and external documentation, Brunton et al. ${ }^{1}$ utilise paternalistic and dehumanising language to describe the population they purport to help. For example, the authors use the term 'subject' to refer to participants and imply a direct association between obesity and disordered eating. ${ }^{1}$ This is especially problematic due to the study's small sample size and the ease through which participants could identify themselves or be identified, resulting in harm from dehumanising labelling. Medical researchers and professionals referring to their patients and human participants in derogatory language in any context is unethical.

Our final concern is that the presented study does not follow the trial protocol registered by the study's authors. ${ }^{29}$ Prospective trial registration is mandated to keep researchers honest. ${ }^{22,30}$ It is intended to ensure that researchers report the outcomes they set out to measure and do not selectively choose those that might show their work in a more positive light. The discrepancies between the registered 
trial protocol approved by the NB-HDEC and the publication are outlined in Table 1.

Amending a trial protocol requires ethics approval and should be reflected in an updated trial registration record. While the authors updated their registration record multiple times, as can be seen in the record history (ACTRN12616001198415), including stating they recruited ten participants, they never amended the intervention period. Furthermore, there has been ample time to follow up with patients since the end of the intervention period (last patient recruited December 2018 according to record). Complete results should be provided to the trial registry one year after completion of the last follow-up. The authors were able to publish a selection of data but have not made complete results available on ANZCTR. Failing to have protocol changes approved and/or selectively reporting research results, whether negligent or intentional, are issues of research integrity. We recognise that data may need to be summarised due to limitations of word count. However, omissions of significant elements of data and protocol changes are not word count issues. The omission of protocol modification information raises a question regarding whether the results have been selectively reported to present the device positively and under-report harms (which may have necessitated a shortening of the study period). It brings the reliability of provided information into question, along with the quality of review by the publishing journal.

There is inherent difficulty in challenging traditional hierarchies of knowledge, and this difficulty is increased when acting as a member of the non-dominant group. The biomedical framing and ethical approval by NB-HDEC lends legitimacy to the research for participants, overriding any concerns they may have had that such a device was demeaning or harmful, because the group tasked with protecting them deemed it 'acceptable'. This prompts critical thought regarding how society enables the dehumanising treatment of fat people. In light of the lack of the above discussion, it is our view that no ethics committee should have granted approval for this study. The lack of scientific rigour, in conjunction with inconsistencies in the registered protocol, should preclude academic publication - and due to the many physical, social and emotional harms experienced by participants in this study, the dental and medical community should not promote the DentalSlim Diet Control.

\section{Table 1 Comparison of trial protocol as reviewed by NB-HDEC and registered (ANZCTR) with the published trial' (QoL = quality of life; IWQoL-} Lite $=$ Impact of Weight on Quality of Life-Lite)

\begin{tabular}{|c|c|c|c|}
\hline Item & $\begin{array}{l}\text { NB-HDEC reviewed and } \\
\text { registered protocol }\end{array}$ & Publication & Comments \\
\hline $\begin{array}{l}\text { Participant } \\
\text { numbers }\end{array}$ & 10 & $\begin{array}{l}\text { Seven recruited, six completed } \\
\text { intervention, but only five } \\
\text { completed the follow-up }\end{array}$ & $\begin{array}{l}\text { From the NB-HDEC minutes (7 June 2016) where project was approved: } \\
\text { - 'The Committee queried what would occur if participants dropped } \\
\text { out, for example } 5 \text { or so, given it is both a tolerability study and the } \\
\text { sample size is only } 10 \text {. The Researcher(s) stated they would plan to } \\
\text { keep trying for } 10 \text { completed, however if vast majority wanted it } \\
\text { removed we would stop the study and re-evaluate it'. } \\
\text { In addition, the trial record history states: } \\
\text { - 'All } 10 \text { participants have been recruited. Updated on 12/12/2018 } \\
\text { 9:46:39 AM'. }\end{array}$ \\
\hline $\begin{array}{l}\text { Intervention aim } \\
\text { and period }\end{array}$ & $\begin{array}{l}\text { Aim of } 10 \% \text { weight loss through } \\
\text { four weeks of liquid diet while } \\
\text { wearing device }\end{array}$ & $\begin{array}{l}\text { Two weeks of liquid diet while } \\
\text { wearing device with two-week } \\
\text { follow-up (reported } 5 \% \text { weight loss) }\end{array}$ & $\begin{array}{l}\text { - No explanation is given in the Brunton et al. (2021) for this } \\
\text { discrepancy } \\
\text { - No amendment was made to the protocol despite other updates } \\
\text { since its original submission } \\
\text { - From the trial registration: 'Instead of eating solid food, the subjects } \\
\text { will be given a liquid diet for } 28 \text { days' } \\
\text { - From the Participant Information Sheet: 'The main aim of the study is } \\
\text { to see how you tolerate wearing the device and how comfortable it } \\
\text { is, and how much weight you can lose in four weeks'. }\end{array}$ \\
\hline $\begin{array}{l}\text { Outcomes } \\
\text { measure } \\
\text { - tolerability }\end{array}$ & $\begin{array}{l}\text { Likert scales measured at } \\
\text { baseline, weekly (for four-week } \\
\text { intervention) and on completion }\end{array}$ & Reported & $\begin{array}{l}\text { - A copy of this questionnaire was not provided in the trial record (as } \\
\text { with other questionnaires) and so whether reporting is complete is } \\
\text { unclear, but more detail is provided here than for other elements. }\end{array}$ \\
\hline $\begin{array}{l}\text { Outcome measure } \\
\text { - oral health }\end{array}$ & $\begin{array}{l}\text { Likert scales at baseline, weekly } \\
\text { (for four-week intervention) and } \\
\text { on completion }\end{array}$ & Not reported & $\begin{array}{l}\text { - One sentence in 'Discussion'. No table of data: 'Surprisingly, few } \\
\text { participants stated that they "occasionally" felt that their oral hygiene } \\
\text { was unsatisfactory or complained about unsatisfactory oral hygiene } \\
\text { post-device removal in this study' } \\
\text { - What 'few' means in a group of } 7 \text { or less (at final follow-up) is unclear. } \\
\text { At least some kind of summary statistic would be the norm. }\end{array}$ \\
\hline $\begin{array}{l}\text { Outcome measure } \\
- \text { QoL }\end{array}$ & $\begin{array}{l}\text { IWQoL-Lite 31-item, five-domain } \\
\text { tool (total score provides } \\
\text { QoL measure) measured at } \\
\text { baseline, weekly (for four-week } \\
\text { intervention) and on completion }\end{array}$ & $\begin{array}{l}\text { Only report items of one domain, } \\
\text { no total domain scores, no total } \\
\text { score }\end{array}$ & $\begin{array}{l}\text { - There is both an IWQOL-Lite ( } 31 \text { items, five domains) and an IWQOL- } \\
\text { Lite-CT for clinical trials ( } 20 \text { items, two domains). The published data } \\
\text { is incomplete for either tool, only describing the physical function } \\
\text { items, with no domain scores and no total IWQoL score. Given the } \\
\text { total score is indicative of QoL, it would be the norm to report at least } \\
\text { the full score, alongside any domains of interest. }\end{array}$ \\
\hline \multirow[t]{2}{*}{$\begin{array}{l}\text { Outcome measure } \\
\text { - weight loss }\end{array}$} & $\begin{array}{l}\text { Baseline, } 24 \text { hours, } 3,7,14 \\
21 \text { and } 28 \text { days during the } \\
\text { planned four-week device use }\end{array}$ & Partial reporting & $\begin{array}{l}\text { - In the publication, the authors report baseline, } 24 \text { hours, } 7 \text { and } \\
14 \text { days, then the intervention stops, then two-week measurement } \\
\text { post-device removal. While the omission of the three-day time point } \\
\text { could be considered acceptable, the discrepancy between protocol } \\
\text { and published intervention periods is worrying }\end{array}$ \\
\hline & One-year follow-up & Not reported & - No explanation provided \\
\hline
\end{tabular}




\section{Ethics declaration}

The authors of this manuscript report no conflict of interests.

\section{Author contributions}

Dr McAllister organised the individuals interested in writing the manuscript. Dr Pausé chaired the group and collated the various writing efforts. All contributing authors participated in the writing, editing and revising of the manuscript. All contributing authors agreed upon the submission to the British Dental Journal.

\section{References}

1. Brunton P A, Ratnayake J, Bodansky H J, Mei L Veerasamy A, Hall R. An intraoral device for weight loss: initial clinical findings. Br Dent J 2021; DOI: 10.1038 s41415-021-3081-1.

2. Pausé C, Taylor SR (eds). The Routledge International Handbook of Fat Studies. London: Routledge, 2021.

3. Hebl M R, Xu J. Weighing the care: physicians' reactions to the size of a patient. Int J Obes 2001; 25: 1246-1252.

4. Warbrick I, Came H, Dickson A. The shame of fat shaming in public health: moving past racism to embrace indigenous solutions. Public Health 2019; 176: 128-132.

5. Gillon A, Pausé C. Kōrero Mōmona, Kōrero ā-Hauora: a Kaupapa Mãori and fat studies discussion of fatness, health and healthism. Fat Stud 2021; DOI: 10.1080/21604851.2021.1906525

6. Hokowhitu B. If you are not healthy, then what are you? Healthism, colonial disease and body-logic. In Tinning R, Fitzpatrick K (eds) Health Education: Critical Perspectives. pp 31-47. Abingdon: Routledge, 2014.

7. Rahiri J-L, Coomarasamy C, MacCormick A, Harwood M, Hill A. Ethnic Disparities in Access to Publicly Funded Bariatric Surgery in South Auckland, New Zealand. Obes Surg 2020; 30: 3459-3465.
8. Faintuch J, Faintuch S. Old and New Anti-obesity Devices for Medical, Surgical, and Endoscopical Use. In Faintuch J, Faintuch S (eds) Obesity and Diabetes: Scientific Advances and Best Practice. pp 959-965. Cham: Springer International Publishing, 2020.

9. Ramsey-Stewart G, Martin L. Jaw wiring in the treatment of morbid obesity. ANZ J Surg 1985; 55: 163-167.

10. Drew P. Weight Loss Surgery Patients' Negotiations of Medicine's Institutional Logic. Res Sociol Health Care 2008; 26: 65-92.

11. Herndon A M. Taking the Devil into Your Mouth: ritualized American weight-loss narratives of morality, pain, and betrayal. Perspect Biol Med 2008; 51: 207-219.

12. Dolezal L, Lyons B. Health-related shame: an affective determinant of health? Med Humanit 2017; 43: 257-263.

13. Lee J A, Pausé C J. Stigma in Practice: Barriers to Health for Fat Women. Front Psychol 2016; DOI: 10.3389/ fpsyg.2016.02063.

14. Prohaska A, Gailey J A. Theorizing fat oppression: Intersectional approaches and methodological innovations. Fat Stud 2019: 8: 1-9.

15. Rodgers $S$, Goss A, Goldney R et al. Jaw wiring in treatment of obesity. Lancet 1977; 309: 1221-1223.

16. Graham R, Hodgetts $D$, Stolte 0 . Dual-heritage households: Food, culture, and re-membering in Hamilton, New Zealand. Int Rev Soc Res 2016; 6: 4-14.

17. Harrop E N, Mensinger J L, Moore M, Lindhorst T. Restrictive eating disorders in higher weight persons: A systematic review of atypical anorexia nervosa prevalence and consecutive admission literature. Int JEat Disord 2021; 54: 1328-1357.

18. Brown-Bowers A, Ward A, Cormier N. Treating the binge or the (fat) body? Representations of fatness in a gold standard psychological treatment manual for binge eating disorder. Health (London) 2017; 21: 21-37.

19. Peat C M, Berkman N D, Lohr K N etal. Comparative Effectiveness of Treatments for Binge-Eating Disorder: Systematic Review and Network Meta-Analysis. Eur Eat Disord Rev 2017: 25: 317-328.

20. Lacey C, Cunningham R, Rijnberg V et al. Eating disorders in New Zealand: Implications for Māori and health service delivery. Int J Eat Disord 2020; 53: 1974-1982.
21. Girz L, Robinson A L, Tessier C. Is the Next Generation of Physicians Adequately Prepared to Diagnose and Treat Eating Disorders in Children and Adolescents? Eat Disord 2014; 22: 375-385.

22. National Ethics Advisory Committee. National Ethical Standards for Health and Disability Research and Quality Improvement. Wellington: Ministry of Health, 2019.

23. World Medical Association. World Medical Association Declaration of Helsinki: Ethical Principles for Medical Research Involving Human Subjects. JAMA 2013; 310: 2191-2194.

24. Emanuel E J, Wendler D, Grady C. What Makes Clinical Research Ethical? JAMA 2000; 283: 2701-2711.

25. Northern B Health and Disability Ethics Committee. Item 16/NTB/89 (CLOSED): Feasibility study of an oral device to promote weight loss. 7 June 2016. Available at https://ethics.health.govt.nz/about-the-committees/ meeting-dates-venues-and-minutes/northern-bminutes/ (accessed July 2021).

26. Tewksbury C, Williams N N, Dumon K R, Sarwer D B Preoperative Medical Weight Management in Bariatric Surgery: a Review and Reconsideration. Obes Surg 2017; 27: 208-214.

27. Livhits M, Mercado C, Yermilov I et al. Does weight loss immediately before bariatric surgery improve outcomes: a systematic review. Surg Obes Relat Dis 2009; 5: 713-721.

28. Holderbaum M, Casagrande D S, Sussenbach S, Buss C Effects of very low calorie diets on liver size and weight loss in the preoperative period of bariatric surgery: a systematic review. Surg Obes Relat Dis 2018; 14 : 237-244.

29. Australian New Zealand Clinical Trials Registry. Sydney (NSW): NHMRC Clinical Trials Centre, University of Sydney (Australia); 2016 - Identifier ACTRN12616001198415 - A Feasibility study to evaluate the effect of a fixed magnetic oral device and liquid diet on weight loss in obese adults. 2016. Available at https://www. anzctr.org.au/Trial/Registration/TrialReview. aspx?ACTRN=12616001198415 (accessed July 2021).

30. Moher D, Hopewell S, Schulz K F et al. CONSORT 2010 explanation and elaboration: Updated guidelines for reporting parallel group randomised trials. Int J Surg 2012; 10: 28-55.

\section{Correction to: An intraoral device for weight loss: initial clinical findings}

The original article can be found online at https://doi.org/10.1038/s41415-021-3081-1.

Author's correction note:

Research article Br Dent J 2021; DOI: 10.1038/s41415-021-3081-1.

When initially published, there were errors in the 'Materials and method' section and Table 1 . In the methods section, 'This study recruited 28 obese volunteer participants with a Body Mass Index (BMI) $>30$ from the wider community in Dunedin, New Zealand' should have read 'This study recruited 28 obese volunteer participants with a Body Mass Index (BMI) $>35$ from the wider community in Dunedin, New Zealand'. In Table 1 , 'BMI $>30$ ' should have read ' $\mathrm{BMI}>35$ '.

In addition, the authors wish to include the following ethics declaration: 'The authors note that, as anticipated by the ethics application, patent protection in the device was sought, with that being done through a new, non-active, holding company in which the research collaborators had proportionate ownership interests'

The authors apologise for any inconvenience caused. 